\title{
Zur Frage nach der Gruppierung der motorischen Bahnen im Pyramidenseitenstrang des Menschen.
}

Von

H. Fabritius, Helsingfors, Finland.

(Mit 1 Abbildung.)

Ein Aufsatz von Kehrer im 41. Bande dieser Zeitschrift, auf den ich erst neulich aufmerksam gemacht wurde, veranlasst mich zu den folgenden Zeilen.

Im Laufe der letzten 5 Jahre hatte ich die verhältnismässig seltene Gelegenheit, 9 Fälle von Stichverletzung des Rückenmarks zu sehen. Meine dabei gemachten Beobachtungen und die im Anschluss daran vorgenommenen umfassenden Literaturstndien lenkten meine Aufmerksamkeit auf einige anscheinend regelmässig bei solchen Verletzungen auftretende Störungen der Motilität, aus denen ich einige Wahrscheinlichkeitsschlüsse auf die Gruppierung der motorischen Bahnen im Pyramidenseitenstrang des Menschen zog. Gegen diese wendet sich nun Kehrer und zwar aus zwei Gründen. Erstens hält er den Versuch für unzulässig, aus den klinischen Erscheinungen irgendwelche Schlüsse auf die Läsion des Markes zu ziehen; das seien alles leider nur "schöne Deduktionen des Beobachters". Zweitens zieht er einen eigenen Fall von diffusem Sarkom des Gehirns mit partiellen Lähmungserscheinungen in einem Arm heran, bei dem die Marchimethode angeblich eine diffuse Verteilung der Degeneration im Pyramidenseitenstrang zeigte.

Zu Kehrers Worten ron den „schönen Deduktionen“ kann ich bemerken, dass sie mich eigentlich gar nicht treffen. Jeder, der auch nur oberflächliche Literaturkenntnis besitzt, wird wissen, dass Versuche, gewisse kardinale Rückenmarkssymptome auf eine Läsion bestimmter Teile des. Querschnitts zurückzuführen, keineswegs von mir stammen, sondern schon seit mehr als 50 Jahren von zahlreichen - auch den nambaftesten - Autoren gemacht wurden. Auf diese Weise sind übrigens äusserst wertvolle Ergebnisse erzielt worden, wie die immer mehr beachteten Arbeiten ron Petrén und die ganze Entwicklung der Lehre von den sensiblen Leitungsbahnen zeigen. Hier 
haben die klinischen und physiologischen Tatsachen zu Schlüssen gezwungen, die nicht nur keine anatomischen Unterlagen hatten, sondern vielmehr mit den anatomischen Kenntnissen in direktem Widerspruch standen. Ich möchte diese Frage ganz kurz streifen, weil sie gerade in diesem Zusammenhang eine grosse prinzipielle Bedeutung hat.

Im Jahre 1878 vermutete Gowers ${ }^{1}$ ), dass die Schmerz- und Temperaturbahnen im Fasc. Gowersi verlaufen. van Gehuchten suchte später diesen Satz mehrmals zu beweisen. 18992) berief er sich auf einen Fall, den Crocq mitgeteilt hatte. Er analysierte ihn genau so, wie ich meine Fälle, und gelangte zu dem Schluss, dass die Läsion bauptsächlich das Gowerssche Bündel betroffen haben müsse. $1907^{3}$ ) veröffentlichte er einen eigenen Fall von Stichverletzung des Rückenmarks, wobei er auf Grund der Symptome detaillierte Angaben (man vergleiche seine Zeichnungen) über die Ausbreitung der Läsion im Mark machte. Minor und Kroll haben in Flatau-Jacobsohns Jahresberichten nicht genug des Lobes für „die äusserst detaillierte und streng wissenschaftliche Kritik aller vorhandenen Symptome". Dabei ist zu bemerken, dass der Fall äusserst verwickelt ist, so dass ran Gehuchten gezwungen ist, eine Menge von Hilfshypothesen anfzustellen. Befremdend ist auch, dass er von den "faisceaux spino-cérébelleaux" als Schmerz- und Temperaturbahn spricht, obwohl ihm ja nicht unbekannt sein kann, dass eine Zerstörung des Cerebellums nicht zu Analgesie führt und das Gowerssche Bündel also nicht die Schmerzbahn darstellen kann. Die klinischen Symptome seiner Patienten mit Rückenmarksverletzungen gelten ihm aber - obwohl er ein vorzüglicher Anatom ist - mehr.

Noch interessanter ist Lewandowskys Standpunkt in dieser Frage. 1904 veröffentlichte er seine grosse anatomische Studie über die Leitungsbahnen des Truncus cerebri usw. Als sensible Wege kommen für ihn hier die Hinterstränge und der Weg über das Cerebellum, der gekreuzt ist, in Betracht. Wäre nun, sagt Lewandowsky, die Lehre Brown-Séquards richtig, so müsste der grösste Teil der Sensibilität durch das Kleinhirn geleitet werden. Da dies jedoch nachweislich nicht der Fall ist, so "stossen wir auf einen noch nicht gelösten Widerspruch zwischen Pathologie und Physiologie" (1. e. S. 75 [135]), und Lewandowsky zögert gar nicht an der Richtigkeit der physiologischen Beobachtungen za zweifeln: Die Brown-Séquardsche Lehre muss falsch sein. Dies gilt für das Tier. „Nun soll es

1) Clinical Society Transactions. 1878. Vol. XI.

2) Semaine médicale. 1899.

3) Neuraxe. Bd. IX. 
aber", sagt Lewandowsky weiter, „beim Menschen anders sein“. Doch bezweifelt er dies: "Wie die Dinge beim Menschen liegen, werden erst weitere Untersuchungen zu zeigen haben" (S. 76).

Drei Jahre später (Funktionen des Nervensystems, 1907) hat er seinen Standpunkt bereits beträchtlich modifiziert. Der gemeinsame Weg für Temperatur und Schmerz kann nur der Seitenstrang sein (S. 207) und „die klinischen Tatsachen machen es wahrscheinlich, dass die Bahn des Temperatur- und Schmerzsinnes eine gekreuzte ist. Zu häufig sind jene Fälle, wo wir neben einer motorischen Parese der einen Seite eine Aufhebung des Temperatur- und Schmerzsinnes der anderen Seite sehen, als dass man sich der hohen Wahrscheinlichkeit dieser Folgerung verschliessen könnte". Um diese Äusserung jedoch etwas einzuschränken, fügt Lewand owsky etwas später zu: „Ehe die Angelegenheit freilich nicht anatomisch und klinisch ganz geordnet ist, bleibt die Annahme immer eine unsichere Hypothese, und Überraschungen sind durchaus noch nicht ausgeschlossen."

Nun kommen wir aber zu seiner letzten Äusserung in dieser Sache (1911 in seinem Handbuch der Neurologie). S. 774 lesen wir: „Eine weitere sekundäre, im Riückenmark selbst kreuzende sensible Bahn ist, wie sich zeigen wird, auf Grund der klinischen Tatsachen völlig sichergestellt1). Die Anatomie hat sie noch nicht aufzuklären vermocht.“ Lewandowsky schlägt vor, diese „im einzelnen anatomisch noch nicht bekannte Bahn" die Brown-Séquardsche Bahn zu nennen.

Einen schöneren Beweis für die Bedeutung der klinischen Erscheinungen für unsere Kenntnis von den Leitungswegen im Rückenmark und ihrer Lage im Querschnitt kann man wohl kaum verlangen. Wir tappen auf dem Gebiet der topischen Rückenmarksdiagnostik nicht so völlig im Dunkeln, wie Kehrer glaubt, denn einige Punkte sind hier schon als fast sichergestellt anzusehen und auf diesen habe ich nach dem Vorgange berühmter Forscher weiter zu bauen versucht. Strümpell sagt bei der Analyse seines in dieser Zeitschrift, Band 23, veröffentlichten bekannten Falles von Stichverletzung des Rückenmarks: „Da alle Qualitäten der Empfindung im rechten Arm erloschen sind, so muss der Schnitt sowohl ..... durchtrennt haben, wobei ich von der durch zahlreiche andere Beobachtnngen höchst wahrscheinlich gemachten Annahme ausgehe "2)" usw.

So viel möchte ich über die prinzipielle Seite der Sache sagen. Was nun meine Fälle betrifft, so geht aus ihnen, d. h. aus ihren

1) Gesperrt von mir.

2) Gesperrt von mir. 
klinischen Erscheinungen zunächst zweifellos eine Tatsache hervor, die von äusserst grosser Bedeutung für unsere Auffassung von der Gruppierung der motorischen Bahnen im Pyramidenseitenstrang ist. Ich meine die Tatsache, die nicht nur in den von mir beschriebenen und zitierten, sondern auch noch in sehr zahlreichen anderen Fällen beobachtet worden ist, dass bei einer Verletzung oder Beschädigung der Pyramidenbahnen einzelne Abschnitte eines Gliedes motorisch gelähmt, andere dagegen verhältnismässig sehr wenig in ihrer Funktion gestört sein können. Gerade ein solcher Fall war es, der zuerst meine Aufmerksamkeit auf diese Frage lenkte. Es war wirklich höchst auffallend, dass der Patient, der im Cervikalmark eine Verletzung hatte, das linke Bein vom Bett erheben and mit guter Kraft Beugung und Streckung des Knies ausführen konnte, dabei aber völlig unfähig war, den Fuss und die Zehen zu bewegen. Wie soll sich nun dies mit der Annahme einer diffusen Gruppierung der motorischen Bahnen im Pyramidenseitenstrang vereinen lassen? Man wird sagen können: Vielleicht liegen hier ähnliche Verhältnisse vor wie im Nervus recurrens, dessen stimmritzenerweiternde Fasern nach dem sog. Rosenbach-Semonschen Gesetz Sehädlichkeiten gegenüber empfindlicher als die übrigen sein sollen. Diese Annahme lässt sich aber auf unsere Frage nicht übertragen. Denn es gibt zahlreiche Fälle von Cervikal- und Dorsalmarkverletzungen, in denen nur Störungen der Hüftbewegungen, dagegen nicht des Fusses vorhanden waren. Mein von Kehrer angegriffener Fall ist dafür ein sehr schönes Beispiel. Die Verletzung lag im oberen Dorsalmark, auf der einen Seite bestand eine totale schlaffe Lähmung, auf der anderen entwickelte sich unter meinen Augen im Laufe von 16-17 Stunden ebenfalls eine Lähmung, die sich aber auf die Hüfte beschränkte. Pat. war mehrere Tage hindurch absolut nicht imstande, Bewegungen der Hüfte auszuführen, wurde aber sein Bein gehoben und der Oberschenkel unterstützt, so beugte und streckte der Patient gut das Knie und konnte in den Fuss- und Zehengelenken alle Bewegungen ausführen. Ähnliche Fälle gibt es nun eine ganze Reibe und von einer Regelmässigkeit im Sinne des Semonschen Gesetzes kann im Pyramidenseitenstrang keine Rede sein.

Es fragt sich dann, wie wir diese grössere Widerstandsfähigkeit einiger Fasern erklären wollen. Warum werden in einigen Fällen die Fasern der Hüftgelenkmuskulatur, in anderen die des Fusses so zu sagen in elektiver Weise verschont? Ich muss auch hier gestehen, dass vorläufig die Annahme einer gruppenweisen, nicht diffusen Anordnung der Pyramidenstrangfasern diese Tatsachen am besten erklärt. 
Hierzu kommen nun folgende auffallende Verhältnisse. Im Grosshirn, in der inneren Kapsel, im Pedunkel und höchst wahrscheinlich auch in der Brücke verlaufen die motorischen Fasern für die verschiedenen Extremitätenabschnitte getrennt. Weiter wissen wir, dass im peripheren Nerven die Fasern für die verschiedenen Muskeln in Gruppen zusammen verlaufen. Medea und Bossi ${ }^{1}$ ) geben neuerlich an (bei der operativen Behandlung der Athetosis durch partielle Resektion der motorischen Nerven), dass die mechanische Erregung uns zu bestimmen erlaubt, „dass die motorischen Nervenfasern für einen einzigen Muskel schon im Nervenstamm in verschiedenen Strängen so zusammen vereinigt sind, dass es möglich ist, sie zu isolieren, zu überpflanzen und in ihrer Funktion zu vernichten". Schliesslich gilt es ja schon als sichergestellt (man vergleiche Henschen in Lewandowskys Handbuch der Neurologie), dass die Fasern sowohl im Opticus und Chiasma wie auch im Tractus in Gruppen zusammen verlaufen, so dass bestimmte Retinabezirke durch verschiedene Teile des Querschnittes der betreffenden Leitungswege repräsentiert werden; ja sogar noch im Grosshirn sollen nach Henschen die Sehfasern nicht diffus durcheinander verlaufen. Wir sehen somit, dass in verschiedenen Gebieten die Nervenbahnen gruppenweise verlaufen, im Rückenmark sollen wir aber mit einem Ausnahmezustand zu tun haben.

Aus dem soeben Gesagten dürfte hervorgehen, dass die Anordnung der Fasern im Pyramidenseitenstrang in Gruppen so, dass zusammengehörige Elemente auch zusammen verlaufen, wenigstens nichts Überraschendes in sich hat. Ausserdem haben wir dann mit der positiven Tatsache zu rechnen, dass bei Pyramidenbahnläsionen isolierte Lähmungen einzelner Abschnitte der innervierten Glieder entstehen können. Ich glaube infolgedessen die Behauptung aufrecht halten zu können, dass $\mathrm{klinis} c \mathrm{~h}$ schwerwiegende Gründe gegen eine diffuse Gruppierung der motorischen Pyramidenbahnen bestehen.

Vor einer viel schwierigeren Aufgabe stehen wir natürlich, wenn wir die Art dieser eventuellen Gruppierung im Pyramidenseitenstrang feststellen sollen. Wenn ich mich an diese Aufgabe heranwagte, so geschah es aus Gründen, die ich hier kurz streifen will, damit sich der nicht orientierte Leser überzeugen kann, inwiefern meine Resultate nur wüste Phantasien sind, wie es Kehrer behauptet.

Ich habe mich auf zwei Kategorien von Stichverletzungen des Ruickenmarks gestützt. Thre Haupttypen wurden schon oben kurz erwähnt.

1) Neurologisches Zentralbl. Juni 1912. 
Ich sah erstens einen Fall mit folgenden Symptomen: Äussere Wunde hinten im Nacken, $1 \mathrm{~cm}$ links von der Mittellinie; Lähmung des linken Armes sowie des Fuss- und Zehengelenkes. Im Knie- und Hüftgelenk dagegen waren die Bewegungen sogar recht gut erhalten. Rechts Thermanästhesie and Analgesie. Bei diesen Befunden ist sicher, 1. dass die Funktionsbeschädigung (oder die Läsion) hier nicht die ganze Pyramidenseitenstrangbahn betroffen haben konnte, 2. dass die nach vorne-aussen von der Pyramidenseitenstrangbahn gelegenen Teile des Markquerschnittes in irgendeiner Weise beschädigt waren, denn sonst hätte eine Analgesie und Thermanästhesie der gekreuzten Seite (die noch 8 Monate später bestanden) nicht vorhanden sein können. Es gibt nun zwei Möglichkeiten: Entweder lag nur ein grösserer Herd im Mark vor; in diesem Falle können wir sagen, dass sogar höhere, wahrscheinlich hauptsächlich die vorderen äusseren Teile der Pyramidenseitenstrangbahn beschädigt waren. Oder es bestanden. mehrere kleine Herde, die uns keine Schlüsse za ziehen erlauben. Zwischen diesen beiden Möglichkeiten ist eine Entscheidung nicht zu treffen.

Ich fand nun in der Literatur drei dem meinen fast völlig gleiche Fälle (Fälle von Vučetić, Boyer, zit. nach Brown-Séquard und Bode). Boyer nimmt auf Grund der Symptome an, dass das Rückenmark lädiert gevresen sein muss, aber, sagt er, „elle n'a du l'être que superficiellement". Bode äussert sich folgendermassen (Berl. klin. Wochenschr. 1891, S. 563): „Es ist somit die mutmassliche Gegend der Läsion in unserem Falle anatomisch ziemlich genau bekannt." Weiter: „Aus der schräg von aussen nach innen verlaufenden Richtung des Stiches erhellt, dass auf alle Fülle die Pyramydenseitenstrangbahn, die Kleinhirnstrangbahn zum Teil, wahrscheinlich aber gar nicht verletzt worden sind“. Ich kann mich somit bei diesen Fällen auf das Urteil der betreffenden Autoren stützen. Bode zieht noch folgenden Schluss auf die Gruppierung der motorischen Bahnen im Pyramidenseitenstrang. Es lag in seinem Falle eine Stichverletzung im rechten Hinterhaupt vor, auf der rechten Seite waren völlig gelähmt M. scalenus, Nerv. phrenicus und der rechte Arm, mit dem rechten Bein „,konnte Patient alle möglichen Bewegungen ausführen“, wenn auch kraftlos. „Diese Erscheinungen gestatten“, schreibt Bode nun, „den ziemlich sicheren Schluss zu ziehen, dass an dieser Stelle die motorischen Leitungsbahnen für die genannten Organe unmittelbar nebeneinander liegen“. Man beachte die Worte Bodes: „ziemlich sicherer Schluss" und vergleiche damit Kehrers Ausdrücke!

$\mathrm{Zu}$ diesen vier Fällen kann ich aus der Literatur noch den folgenden sehr interessanten Fall fügen: 
Der Fall stammt von Rieck ${ }^{1}$ ). Äussere Wunde etwas links von der Mittellinie in der Höhe des D3-4. Am folgenden Tag vermag Patient „das linke Bein nur durch den. Ileopsoas emporzuwerfen, aber kann es nicht erhoben halten. Der Unterschenkel konnte dabei nicht gestreckt werden, fiel schlaff herunter. Auch Bewegungen des Fusses und der Zehen sind unmöglich". Rechts bestand ungestörte Motilität, aber Thermanästhesie und Analgesie.

Ich möchte wirklich wissen, wie man diesen Fall durch die Annahme einer diffusen Verteilung der Fasern der Pyramidenbahnen erklären will. Den Fuss und die Zehen kann der Patient nicht bewegen, wohl aber das Bein emporwerfen. Müssen wir nicht hier, ebenso wie Bode in seinem Fall, den "ziemlich sicheren Schluss" ziehen, dass die Bahnen des Ileopsoas nebeneinander liegen und von der Verletzung verhältnismässig weniger als die übrigen Bahnen betroffen wurden? Wo aber liegen sie denn im Querschnitte? Das mag unentschieden bleiben! Vorläufig wollen wir nur feststellen, dass die motorischen und die sensiblen Symptome, und die Lage der äusseren Wunde in allen fünf besprochenen Fällen völlig übereinstimmten. Wir haben einen klinischen Typus von Stichverletzung des Rückenmarks vor uns, dessen Unterlage von uns noch als ein Rätsel angesehen werden mag.

Nun kommen wir aber zum zweiten Typus, zur zweiten Kategorie von Stichrerletzungen des Rückenmarks, auf die ich mich berufen habe. Den Typus dieser Kategorie stellt mein von Kehrer ganz besonders angegriffener Fall vor. Der Patient wurde im Dorsalmark verletzt. Die äussere $W$ unde liegt etwas links von der Mittellinie, das rechte, also das gegenüberliegende Bein wird gelähmt, links besteht eine totale Anästhesie. Die Motilität auf dieser Seite, also im linken Bein, war 17 Stunden nach der Verletzung insofern von der Norm abweichend, als Patient das Bein nur mit Mühe aufheben konnte. Im Knie und Fuss dagegen bestand gute Beweglichkeit. Noch 6-7 Stunden später war die linke Hüfte völlig gelähmt, das Knie und der Fuss wurden aber noch immer gut bewegt. Hier ist sicher, dass der rechte Seitenstrang nicht funktionierte, da wir sonst keine totale lähmung des rechten Beins und keine Aufhebung der Schmerz-Temperaturempfindlichkeit links gehabt hätten. Ganz abgesehen von der Ursache der Symptome würde nun jeder Fachmann die Diagnose: Hempilegia spinalis dextra stellen, also einen grösseren oder kleineren Herd rechts annehmen. Nun sehen wir, dass im Laufe von etwa 20-24 Stunden nach der Verletzung auch eine linksseitige Läh-

1) Rieck, Über Halbseitenläsion des Rückenmarks. Diss. Berlin 1897. 
mung eintritt, aber nur im Hüftgelenk. Liegt es nun nicht näher anzunehmen, dass sich von dem rechts sicher vorhandenen Herde schädigende Einflüsse auf die linke Markhälfte ansgebreitet haben, als dass irgendwelche anderen undefinierbaren und nicht einmal vermutnngsweise anzugebenden Umstände obgewaltet haben. Ich habe mich für die erste Annahme entschieden und also angenommen, dass die Bahnen für die Hüfte mehr nach innen im Pyramidenseitenstrang liegen, was mit den Erfahrungen aus den fünf früheren Fällen gut übereinstimmt.

In der Literatur fand ich sieben weitere Fälle, in denen völlig mit den soeben beschriebenen analoge Verhältnisse herrschten. Einer von diesen Fällen, der Fall von Müller ist ein Sektionsfall. Die Mark-

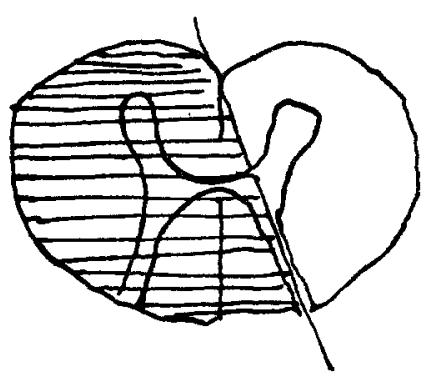

Fig. 1. verletzung stimmte hier völlig mit dem überein, was man aus den Symptomen erschliessen konnte. Die äussere Wunde lag rechts von der Mittellinie in der Höhe des D4. Das linke Bein war völlig gelähmt, rechts bestand totale Anästhesie. Am 4.-5. Tage trat auch Lähmung des rechten Beins auf, am folgenden (6.) Tage aber besserte sich der Zustand „so weit, dass die Kranke Zehen und Fall Müller.

Fuss rechts bewegen kann". Die Sektion zeigte folgendes Bild (Fig. 1):

Natürlich sagt uns diese Sektion auch nichts ganz Gewisses über die Ursachen der Lähmungserscheinungen auf der rechten Seite aus, denn auf einen Fall allein darf man sich nicht stützen. Aber in ihrer Gesamtheit bilden die Fälle, die ich angezogen habe, doch etwas anderes als nur die Grundlage für eine „blosse schöne Deduktion."

Diese Fälle stellten mein Hauptbeweismaterial dar (ausserdem hatte ich noch mehrere Fälle, die keine Stichverletzungen des Markes waren, zusammengestellt). Nur der Fall Rieck ist neu. Zwei weitere neue Fälle möchte ich nun hier hinzufügen.

Der eine ist der alte und bekannte, oben kurz erwähnte Fall von Gowers, den er schon 1878 beschrieb: Ein junger Mann will Selbstmord verüben und schiesst sich zu diesem Zwecke eine Kugel in den Mund. Es stellte sich sofort eine totale rechtsseitige Lähmung ein. Am folgenden Tage bewegte Pat. aber das Bein wieder, während der Arm gelähmt blieb. Der Exitus trat am dritten Tage ein. Bei der Sektion fand man die Kugel zwischen den Bögen des Atlas und des Epistropheus. Ein kleiner Knochensplitter hatte die Dura rechts vorne durchbohrt und "was adherant to the surface of the cord". Eine 
mikroskopische Untersuchung scheint nicht gemacht worden zu sein. Es heisst nur, dass sich im rechten Seitenstrang zahlreiche Blutungen fanden, Hinterstrang und Hinterhorn dagegen ziemlich intakt waren. Nun ist die Frage, weshalb der Pat. am Tage nach der Verletzung das rechte Bein, nicht aber den Arm bewegen konnte. Diese Erscheinung muss höchst auffallend gewesen sein, ganz wie bei meinem ersten Fall oben, in dem der Pat. Hüfte und Knie, nicht aber Fuss und Zehen bewegte - denn Gowers widmet ihr grosse Aufmerksamkeit und sucht sie zu erklären. Er denkt an zwei Möglichkeiten. Entweder sind einige unter den Pyramidenseitenstrangbahnen unbeschädigt geblieben oder es hatte eine Kompensation von der gesunden Seite stattgefunden. Gowers hält das letztere für wahrscheinlicher, indem er sich vorstellt, dass einige Pyramidenbahnfasern, und zwar diejenigen des rechten Beines, noch ungekreuzt waren (in der Höhe von C 2!) und somit der Beschädigung entgingen.

Diese Annahme lässt sich wohl kaum aufrecht erbalten. Unsere jetzige Erfahrung zeigt doch, dass die Motilität beim Menschen nicht am folgenden Tage zurückkehrt, wenn eine Lähmung infolge einer totalen Funktionsaufhebung des einen Seitenstranges eingetreten ist. Vor allem aber können wir folgenden Einwand machen. Wenn bei Gowers' Patient die Bahnen des rechten Beines in erheblichem Grade in der Höhe von $\mathrm{C} 2$ ungekreuzt, d. b. auf der linken Seite verlaufen wären, so bätte dies auch für die Bahnen des linken Beins der Fall sein und so eine Parese wenigstens links bestanden haben müssen. Dies war aber nicht der Fall.

Man könnte vielleicht aber annehmen, dass die Bahnen des Beines widerstandsfähiger seien als die des Armes; da nun im Falle Gowers' keine tatsächliche Durchtrennung des ganzen rechten Seitenstrangs vorlag (nur vorn rechts), so wäre es auch bei einer diffusen Verteilung der Fasern im Pyramidenseitenstrang' verständlich, dass die Beweglichkeit im Bein erhalten geblieben bzw. am Tage nach der Verletzung zurückgekehrt ist. Nun gibt es aber einen von Hilbert 1) mitgeteilten Fall (Fall II bei Hilbert), der in schroffem Gegensatz zu dieser Annahme steht.

Es handelt sich um einen Fall von Stichverletzung. Die äussere Wunde war im Nacken, links von der Mittellinie an der Haargrenze. Es bestand vollständige Lähmung des rechten Armes und Beines, das linke Bein war paretisch, der Arm aber konnte ziemlich gut bewegt werden. Die Parese des linken Armes ging allmählich zurück. Rechts blieben die Lähmungen lange bestehen.

1) Deutsche med. Wochenschr. 1906. S. 1220. 
Hier haben wir ein Beispiel aus der ,zweiten Kategorie" der obigen Fälle. Die totale und schwere lähmung liegt auf der der änsseren Wunde gegenüberliegenden Seite. Hier, in der rechten Markhälfte also, muss irgendein Herd vorhanden gewesen sein; das zeigt erstens die schwere und lang dauernde Lähmung, zweitens eine Angabe Hilberts, dass der Stichkanal schräg von links nach rechts auf die Wirbelsäule zu verlief. Weiter zeigte eine Röntgenphotographie, dass der Dornfortsatz des dritten Halswirbels durchtrennt war. Spricht nun nicht alles dies dafür, dass die in der linken Markhälfte vorhandene Störung, die die Parese des linken Beins verursachte, in dem Gebiete des Querschnitts lag, das dicht an die in der rechten Markhälfte sicher beschädigten Teile grenzte? Oder sollen wir wieder irgendwelche neuen Erklärungen für die vorhandenen Symptome suchen? Jedenfalls können wir diesen Fall nicht in derselben Weise wie den Fall Gowers verstehen; die Läsion liegt auch hier hoch oben im Cervikalmark, wir haben es aber hier mit einer auf Pyramidenbahnenverletzung beruhenden Motilitätsstörung im Bein zu tun bei verhältnismässig intaktem Verhalten des Armes, während es sich im Fall Gowers um eine Lähmung des Armes bei gleichzeitig vorhandener Motilität des Beines handelt.

Wo liegt nun die Erk] örung für alle die oben mitgeteilten Erscheinungen? Oder vielleicht gibt es überhaupt keine andere Erklärung, als dass es sich um „Zufälligkeiten“, um ,individuelle Verhältnisse" u. dgl. handelt. Mir scheint noch immer, dass die ron mir gegebene Erklärung jedenfalls ernsthaft in Betracht gezogen werden muss.

Natürlich gibt es ja - und das habe ich in meinen früheren Arbeiten mehrmals hervorgehoben - Fälle, die von den obigen stark abweichende Verhältnisse zeigen. Aber dies ist ja schliesslich kein Wunder, sondern muss im Gegenteil als selbstrerständlich exwartet werden. Die Beobachtungen lassen leider nicht selten viel zu wünschen übrig, vor allem muss man aber schon aus rein theoretischen Gründen voraussehen können, dass eine den obigen Beschreibungen entsprechende Form der Lähmung nur verhältnismässig selten auftreten kann. Eine gewisse Gruppierung der Bahnen im Pyramidenseitenstrang angenommen, können wir darum doch nicht verlangen, dass sie nebeneinander stehen sollen, wie die Soldaten in einer Reihe, und ebensowenig können wir voraussetzen, dass sich die funktionsschädigenden Einflüsse immer wie die Wellen am Strande in regelmässiger Form uiber den Markquerschnitt ausbreiten. Wir können uns ja gut vorstellen, dass die Läsion die motorischen Fasergruppen sozusagen von der Seite angriffe. In diesem Falle würden wir aber keine isolierten 
Monoplegien erwarten dürfen, sondern eine diffuse Parese sämtlicher Abschnitte eines Gliedes.

Schliesslich könnte sich noch ein Einwand gegen eine gruppenweise Anordnung der motorischen Bahnen aufdrängen. Man wird sagen können, dass es sich beim Pyramidenseitenstrang um ein zu kleines Gebiet handelt, also dass eine partielle Beschädigung durch eine äussere Stichverletzung nicht zustande kommen könne, das erforderliche verletzende Instrument sei dazu viel zu grob.

Dieser Einwand ist jedoch nicht stichhaltig. Der oben kurz referierte Fall Müller (s. Fig. 1) lehrt uns, dass eine Messerklinge, obwohl sie in unmittelbarer Nähe der Pyramidenbahn im Mark steckt, dennoch keine Lähmungserscheinungen hervorzurufen braucht. Das rechte Bein der Patientin war 4 Tage nach der Verletzung motorisch intakt, obwohl das Messer dicht an der medialen hinteren Seite des rechten Pyramidenseitenstranges lag. Noch lehrreicher ist der Fall Klare. Ein Mann wurde im Rücken durch einen Messerstich verletzt; er geht selbst ins Spital, wo er verbunden und sofort wieder entlassen wird. Eine Woche später kommt er zurück, und man zieht ein $6 \mathrm{~cm}$ langes Stück einer abgebrochenen Messerklinge aus der Wunde heraus; es nützt aber nichts mehr und der. Pat. stirbt an Meningitis. Die Sektion zeigte, dass das Rückenmarlz in der Höhe des 4. Brustwirbels von hinten nach vorne in seiner Mitte durchbohrt war. Diese Fälle zeigen also unzweideutig, dass die Wirkung einer Stichverletzung auf die nächstbenachbarten Teile des Rückenmarks äusserst gering, ja fast gleich Noll sein kann. Somit scheint mir, können wir auch verstehen, dass bei einer Stichverletzung nur eine partielle Beschädigung der Pyramidenbahn auftreten kann.

Dies ist nun das Material, das ich zur Beleuchtung und Verteidigung meines Standpunktes heranziehen kann. Gegen ihn hat sich Ke hrer, wie oben gesagt, gewandt. Er berichtet auch einen eigenen Fall von cerebraler Monoplegie und beruft sich ausserdem auf zwei andere ähnliche Fälle aus der Literatur, in denen eine diffuse Faserdegeneration im Pyramidenseitenstrang vorhanden war.

Gegen die Verwertung dieser cerebralen Fälle lassen sich aber Bedenken erheben.

Es ist ja eine bekannte Tatsache, dass auch bei totaler Unterbrechung der Pyramidenbahn in der inneren Kapsel keine totale, sondern nur eine fleckweise Degeneration des Pyramidenseitenstranges auftritt. Die grosse Autorität auf diesem Gebiete, v. Monakow, schreibt darüber in seiner Hirnpathologie (2. Anfl.) S. 1140: „Es ist hervorzuheben, dass, selbst wenn die Unterbrechung der Pyramidenbahn in der inneren Kapsel eine radikale war, im korrespondierenden 
Seitenstrangfelde nie sämtliche Nervenfasern dem Untergange verfallen, sondern dass degenerierte und normale Fasern nebeneinander und oft innig gemischt sich rorfinden. Es lässt sich dies wohl so erklären, dass das Feld der Pyramidenseitenstrangbahn auch eine Menge anderer zur Pyramide in keiner Beziehung stehender Nerrenfasern enthält." Diese Verhältnisse müssen wohl offenbar in hohem Maße die Beurteilung eines Degenerationsfeldes erschweren, das durch einen cerebralen Herd im Areal der Pyramidenbahn im Rückenmark verursacht wird, und dies um so mehr, als noch eine zweite Fehlerquelle in Betracht gezogen werden muss. Henschen macht bei seiner oben kurz erwähnten Besprechung der Gruppierung der Fasern im N. opticus darauf aufmerksam, dass bei alten Atrophien eine Verlagerung der atrophischen Felder stattfinden kann. "An alten Atrophien", schreibt er (Lewandowskys Handbuch der Neurologie, allgemeiner Teil, S. 900), ,werden diese Felder narbenförmig verlagert und die erhalten gebliebenen Bündel in die atrophischen Felder hineingezogen; daher wohl die Verwirrung in den Anschauungen." Ich glaube, diese letzte Bemerkung Henschens hat auch für unsere jetzt zu behandelnde Frage einige Berechtigung.

So viel möchte ich über alte Fälle von cerebraler Monoplegie sagen. Gehen wir nun zu frischen Fällen über, also zu Fällen, in denen die Marchi-Methode in Betracht kommt, so müssen wir uns auch hier fragen, ob sie für unsere vorliegende Frage so geeignet sind, wie Kehrer meint. „Aufschlüsse in dieser rein topographischen Frage", sagt er, "kann beim Menschen nur das Studium der sekundären Degeneration, vor allem nach Marchi, geben." Dieser Äusserung gegenüber möchte ich mich wieder auf $\nabla$. Monakow berufen. Es ist ganz auffallend, dass er so oft und bei verschiedenen Gelegenheiten auf die Mängel der Marchi-Methode für die Entscheidung feinster anatomischer Details hinwejst. Im Archiv für Psychiatrie, Bd. 31, S. 68, sagt er anlässlich einiger Resultate Tschermaks: „Seine Untersuchung führte T. unter Anwendung der MarchiFärbung aus. Wie ich schon früher betont habe, werden durch diese Methode nur die Fettschollen gefärbt, diese haften aber der degenerierten Faser nicht lange an; sie sind vielmehr in hohem Grade wanderungsfähig. Man findet die schwarzen Körnchen oft in Hirnregionen von operierten Tieren, wo die Nervenelemente normal sind. Za welchen unrichtigen Schlüssen man bei Anwendung der MarchiMethode gelangen kann, zeigt u. a. die Arbeit von Giuglianschi, der nach Querdurchtrennung des Lendenmarks beim Hunde eine Massenansammlung von schwarzen Schollen in der Umgebung des Zentralkanals fand und hieraus auf das Vorhandensein eines weissen. 
Faserzuges in der Gegend zwischen Zentralkanal und Hinterstrang schloss." In seiner Hirnpathologie äussert sich v. Monakow in ähnlicher Weise. Sogar in dem grossen Übersichtsreferat über den gegenwärtigen Stand der Hirnlokalisation benutzt er die Gelegenheit, um vor den Resultaten "der leicht irreführenden Marchi-Methode" zu warnen. - Durch alle diese Äusserungen wird natürlich der Wert der Marchi-Methode nicht vernichtet, ich glaube aber, dass sie gerade hier der kategorischen Ausdrucksweise Kehrers gegenüber am Platze sind. „Nur das Studium der sekundären Degeneration und vor allem nach Marchi“, sagt Kehrer, „kann unsere Fragen lösen“; das Obengesagte dürfte doch einige Bedenken gegen die unbedingte Gültigkeit dieser Worte aufkommen lassen.

Schliesslich möchte ich noch kurz auf die neulich erschienene Arbeit von Höstermann (Arch. f. Psych. Bd. 49) hinweisen. Er stellt eine ganze Reihe von Fällen zusammen, in denen trotz hochgradiger Erkrankungen der einen Hemisphäre und Lähmung der entsprechenden Extremitäten keine Degeneration im Pyramidenseitenstrang nachzuweisen war; der von Binswanger beschriebene Fall soll sogar Lähmung und intakte Pyramidenbahn bei Fehlen von Riesenpyramidenzellen gezeigt haben. Rondoni wiederum fand bei Idiotengehirnen Fehlen der Pyramidenriesenzellen bei intakter Pyramidenbahn und erhaltener Motilität. Ich habe selbst neulich einen Fall gesehen, eine von Kindheit an epileptische Frau, die im Alter von 53 Jahren starb. Die 10 letzten Jahre ihres Lebens war sie links gelähmt; das Gewicht der linken Hemisphäre betrug $560 \mathrm{~g}$, das der rechten $290 \mathrm{~g}$. Degenerationen waren weder im Pons noch im Rückenmark nachweisbar. Wir stehen somit vor einer Reihe von Tatsachen, die uns mahnen, aus cerebralen Erkrankungen nur mit grosser Vorsicht detaillierte Schlüsse in Bezug auf die Pyramidenbahn zu ziehen.

Was nun schliesslich den von Kehrer beschriebenen Fall betrifft, so lässt seine Beweiskraft wirklich viel zu wünsehen übrig. Die Krankheitsgeschichte steht erstens in schroffem Widerspruch zu Kehrers Besprechung. Hier sagt er: „Worauf es aber besonders ankommt, das ist die Tatsache, dass mindestens 28 Tage ante exitum eine völlige Lähmung der Fingerstrecker links bestand, die sich nach einem Rindenkrampf 15 Tage später auf die Strecker und Pronatoren der Hand ausdehnte." In der Krankengeschichte finden wir aber folgende (kurze, knappe) Angaben:

D. 29. IX.08 (bei der Aufnahme): „Rohe Kraft im Arm links gering. Beim Strecken der linken Hand bleibt der 3.-5. Finger deutlich zurück."

13. X. 08. „Kann die linke Hand nicht völlig strecken, am wenigsten den Mittelfinger. Nachts klonische Zuckungen in Hand- und Ellenbogen- 
gelenk mit Bewegungsverlust. Danach Lähmung; Unfähigkeit, die Hand und den 3.-5. Finger zu strecken und $\mathrm{zu}$ pronieren."

26. X. 08 Exitus.

Nach der Krankengeschichte lag also 28 Tage vor dem Tode keineswegs eine „,ö̈llige Lähmung“ der Fingerstrecker links, sondern eine Parese im linken Arm vor; beim Strecken der 1. Hand bleibt nur der 3.-5. Finger "deutlich" zurück. Noch 14 Tage später also 14 Tage vor dem Tode - besteht keine völlige Lähmung dieser Strecker, sondern erst jetzt tritt, wie die Krankengeschichte ausdrücklich sagt, „Unfähigkeit, die Hand und den 3.-5. Finger zu strecken und pronieren" ein. Die motorischen Symptome sind hier somit nicht eng begrenzt und deutlich, und ebensowenig ist der Sektionsbefund eindeutig. Es wurde ein diffuses Sarkom im Gehirn gefunden - die klinischen Symptome waren auch sonst so undeutlich, dass die Beobachter "fast bis zuletzt mehr geneigt waren, eine progressive Paralyse mit galoppierendem Verlauf anzunehmen". Aus alledem geht somit hervor, dass wir keineswegs behaupten können, die im Rückenmark nach Marchi sichtbaren Schollen entsprächen gerade den Bahnen der Fingerstrecker. Es kann der Einwand nicht zurückgewiesen werden, dass nicht auch einzelne andere Fasern durch das diffuse Sarkom zerstört wurden und dass diesen einzelne von den spärlichen Fettschollen im Rückenmarksquerschnitt entsprachen. Der Fall berechtigt nicht zu sicheren Schlüssen.

Fassen wir die obigen Ausführungen kurz zusammen, so muss zunächst gesagt werden, dass die Frage nach der Gruppierung der motorischen Bahnen im Pyramidenseitenstrang des Menschen wohl nicht als gelöst angesehen werden kann.

Einerseits gibt es zahlreiche klinische Beobachtungen (Stichverletzungsfälle, Tumoren, Myelitis usw.), die uns bei einer Beschädigung der Pyramidenbahn im Rückenmark eine nur partielle Lähmung in dén abhängigen Teilen zeigen, also eine Lähmung z. B. in einem. Gelenk eines Gliedes, bei verhältnismässig guter Motilität in den übrigen Abschnitten. Diese Tatsache lässt sich mit der Annahme einer diffusen Verteilung der Bahnen nur sehr schlecht vereinigen. Auch würde diese Annahme den motorischen Bahnen im Rückenmark gewissermassen eine Sonderstellung einräumen. In der inneren Kapsel, im Pes pedunculi und wahrscheinlich auch im Pons verlaufen diese Bahnen in Gruppen zusammen, im N. opticus verlaufen Bündel zusammengehöriger Fasern bündelweise zusammen, und anch in peripheren Nerven liegen die Fasern der verschiedenen Muskeln beieinander.

Andererseits gibt es einige wenige Fülle (vor allem den Fall 
Zur Frage nach der Gruppierung der motorischen Bahnen usw. 239

Gierlich), in denen klinisch mehr oder weniger ausgesprochene Monoplegien vorhanden waren, als deren Ursache cerebrale Herde aufgefunden wurden. Im Rückenmark konnte hierbei kein zusammenhängendes Degenerationsfeld. festgestellt werden, sondern es fanden sich nur unregelmässig zerstreute Flecke.

Eine einigermassen befriedigende Antwort auf diese Frage wird man m. E. erst dann erwarten können, wenn es gelingt, einige Fälle von frischen Rückenmarksverletzungen oder -Erkrankungen mit partiellen Lähmungserscheinungen von dem oben geschilderten Typus auch post mortem genau zu untersuchen. 